\title{
Enseigner en anglais dans les universités françaises: quels effets prévisibles?
}

\section{Claude Truchot}

\section{(2) OpenEdition \\ Journals}

Édition électronique

URL : http://journals.openedition.org/esp/2792

DOI : 10.4000/esp.2792

ISSN : 2532-0319

Éditeur

Centre d'Information sur l'Éducation Bilingue et Plurilingue

\section{Édition imprimée}

Date de publication : 1 décembre 2013

Pagination : 87-90

ISSN : 1127-266X

\section{Référence électronique}

Claude Truchot, «Enseigner en anglais dans les universités françaises: quels effets prévisibles ? »

Éducation et sociétés plurilingues [En ligne], 35 | 2013, mis en ligne le 01 février 2020, consulté le 17

février 2021. URL : http://journals.openedition.org/esp/2792 ; DOI : https://doi.org/10.4000/esp.2792 


\section{ENSEIGNER EN ANGLAIS DANS LES UNIVERSITÉS FRANÇAISES: QUELS EFFETS PRÉVISIBLES?}

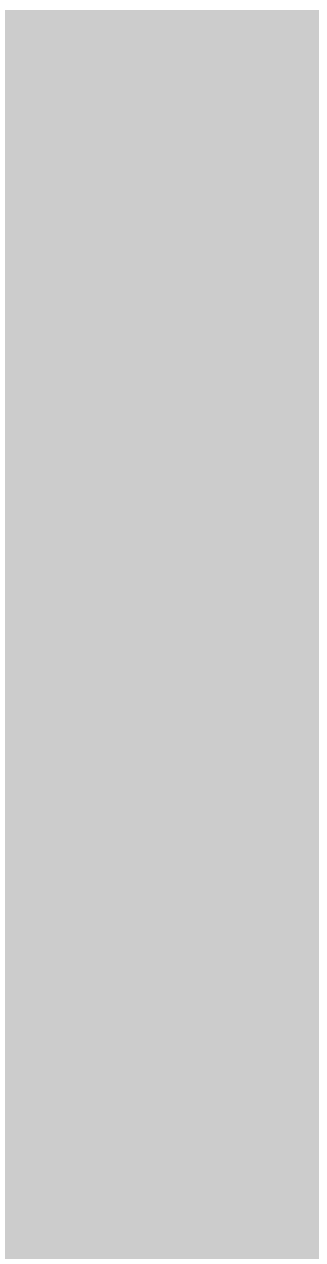

\section{Claude Truchot}

O omme la proposition de loi relative à l'attractivité universitaire de la France, un projet de loi d'orientation de l'Enseignement supérieur et de la Recherche propose de rompre avec le principe qui fait du français la langue de l'enseignement, des examens, des concours et des thèses. Ce principe est inscrit dans la loi de 1994, dite loi Toubon, et découle de l'article 2 de la Constitution selon lequel «La langue de la République est le français». Une partie des enseignements effectués dans le cadre d'accords avec des universités étrangères ou de programmes financés par l'Union européenne pourrait ainsi être dispensée en langues étrangères, ce qui veut dire en anglais. Dans la mesure où tous les cursus et diplômes à caractère international incluent nécessairement ces partenariats, et où toutes les formations universitaires ont vocation à être internationales, le champ ainsi ouvert à l'enseignement en anglais est donc vaste.

On aurait pu s'attendre à ce que les effets prévisibles d'un tel projet aient fait l'objet d'études préalables et d'évaluations par les autorités publiques compétentes. Or il n'en est rien. Pourtant on peut se référer à de nombreuses références instructives puisque l'enseignement en anglais s'est très largement répandu en Europe du Nord, et qu'il a été développé en Allemagne depuis une dizaine d'années. Sur l'Europe du Nord on dispose d'études et de témoignages que j'ai présentés dans diverses publications (1) et sur l'Allemagne une véritable évaluation a été réalisée par la Conférence des chefs d'établissements d'enseignement supérieur, Hochschulrektoren-conferenz, c'est-à-dire l'équivalent en France à la fois de la Conférence des présidents d'université et de la Conférence des grandes écoles (2).

En Europe du Nord, le recours à l'anglais a été un moyen de compenser la faible diffusion internationale des langues nationales, considérée comme un handicap pour l'attractivité internationale des universités, notamment lors de la mise en place du programme d'échanges universitaires Erasmus qui a vu les étu- 
Enseigner en anglais dans les universités françaises: quels effets prévisibles?

C. Truchot diants se tourner en masse vers les universités britanniques, françaises et espagnoles. Pour ce faire, les universités nordiques et néerlandaises se sont appuyées sur une compétence acquise: la connaissance de l'anglais. Ces universités on ainsi pu attirer une proportion plus importante d'étudiants étrangers. Précisons toutefois que celle-ci reste très largement inférieure à ce qu'elle est en France. Par contre, les effets négatifs sont multiples et de plus en plus mis en évidence.

- Les langues nationales disparaissent des enseignements de haut niveau. Aux Pays-Bas, les masters tendent à avoir lieu presque exclusivement en anglais. Ainsi, en 2008-2009 à l'université de Maastricht, sur 46 masters le seul qui n'était pas en anglais était celui de droit néerlandais.

- Calqués sur les enseignements dispensés par les universités américaines, les cursus perdent toute spécificité et ignorent les travaux scientifiques européens.

- Aux Pays-Bas, les universités ont perdu non seulement l'usage du néerlandais, mais aussi l'ouverture internationale qui les caractérisait traditionnellement par la connaissance d'autres langues surtout l'allemand et le français, actuellement ignorées des étudiants.

- Si le niveau de connaissance de l'anglais parmi les enseignants est généralement considéré comme correct, l'usage qu'ils peuvent en faire n'est pas celui qu'en ferait un anglophone. Les cours sont récités, les échanges avec les étudiants sont limités et ont lieu souvent dans une langue approximative, la perte d'information dans la transmission des connaissances est considérable.

Le bilan probable - mais les autorités n'ont jamais osé le faire est que la qualité de l'enseignement supérieur a baissé dans ces pays.

Cette prise de conscience s'est faite en Allemagne à la suite de dix ans d'anglicisation. Le bilan par les chefs d'établissements est sans concession.

- S'ils admettent que l'enseignement en anglais a pu renforcer l'attractivité de certains cursus, ils constatent qu'ils attirent des étudiants étrangers dont le niveau en anglais est insuffisant. De nombreux enseignants allemands n'ont pas «les compétences linguistiques requises pour garantir que l'enseignement dispensé en anglais soit de haut niveau ".

- La polarisation sur l'anglais se fait au dépend des autres langues et de l'ouverture internationale des universités.

- La mise à l'écart de l'allemand des enseignements internationaux contrecarre les efforts faits pour développer la diffusion et le prestige de cette langue dans le monde. 
Enseigner en anglais dans les universités françaises: quels effets prévisibles?

C. ТRUснот
- Alors que les échanges internationaux devraient permettre aux étudiants, chercheurs et enseignants venant de l'étranger de connaître la culture et apprendre la langue du pays qui les accueille, ici l'allemand, cet aspect est marginalisé.

- Ce mode d'internationalisation crée de multiples problèmes de gestion auxquels les universités ne peuvent faire face.

En lieu et place de l'anglicisation, les chefs d'établissements prennent position en faveur d'une ouverture internationale fondée sur des politiques linguistiques d'universités. La Recommandation qu'ils ont adoptée en novembre 2011 assigne à ces politiques un double objectif, celui de préserver le rôle de l'allemand comme langue d'expression et de transmission des savoirs et celui de garantir une formation linguistique diversifiée et de niveau élevé, incluant la formation à l'allemand des chercheurs et étudiants étrangers, la connaissance de l'anglais mais aussi d'autres langues.

En somme, on s'apprête à faire en France ce qui a été fait en Allemagne il y a dix ans et ce à quoi les responsables universitaires allemands tournent résolument le dos actuellement, après un constat très négatif des conséquences qu'ils ont pu mesurer.

On pourrait ajouter que si les pays nordiques, les Pays-Bas, l'Allemagne ont estimé pouvoir miser sur anglais, et constatent actuellement ses limites, un constat préalable réaliste est qu'en France on n'est pas en compétence de miser sur cette langue.

Par contre, on a celle de miser sur le français, de recruter dans cette langue en s'appuyant sur des réseaux internationaux bien organisés et constitués à l'échelle planétaire, de garantir aux étudiants et chercheurs étrangers une formation en français grâce à une compétence reconnue dans l'enseignement du français langue étrangère et seconde (FLE/FLS). Ce que les établissements d'enseignement supérieur devraient offrir en plus, qui ferait la différence, et que n'offrent pas les universités anglophones, ni les universités nordiques, c'est une formation approfondie et diversifiée en langues étrangères. C'est dans le développement de cette compétence plurilingue qu'il faudrait investir. On ne contribuera pas à redresser l'économie française en faisant bafouiller dans l'enseignement supérieur un anglais de composition, mais en valorisant nos ressources linguistiques, en ouvrant nos universités et nos étudiants à des langues qui permettent de nouer des partenariats solides et durables, celles des autres pays, et en premier lieu celles de nos partenaires les plus proches,

Il serait mieux pour une ministre de l'enseignement supérieur et 
Enseigner en anglais dans

les universités françaises:

quels effets prévisibles?

C. ТRUснот de la recherche d'attacher son nom à une initiative linguistique novatrice qu'à un projet suiviste et dépassé.

\section{NOTES}

(1) Europe: l'enjeu linguistique, Paris: La documentation française, Collection Études, 160 pages, «L'enseignement supérieur en anglais véhiculaire, la question de la qualité», revue de géopolitique diploweb.com, http://www.diploweb.com/L-enseignement-superieur-en.html

(2) Hochschulrektorenconferenz, Politique linguistique dans les universités allemandes. Recommandation de la XIe assemblée plénière du 22 novembre 2011 à Berlin. http://www.hrk.de/positionen/beschluesse-nach-thema/convention/empfehlung-sprachenpolitik-andeutschen-hochschulen/ 